\title{
V. On the true and extended interpretation of formulæ in spherical trigonometry
}

\section{James Thomson LL.D.}

To cite this article: James Thomson LL.D. (1837) V. On the true and extended interpretation of formulæ in spherical trigonometry , Philosophical Magazine Series 3, 10:58, 18-24, DOI: 10.1080/14786443708649064

To link to this article: http://dx.doi.org/10.1080/14786443708649064

册 Published online: 01 Jun 2009.

Submit your article to this journal $\sqsubset \pi$

Џll Article views: 2

Q View related articles $₫$ 
were solid previously to their elevatory movements; and that they also possessed lines of structure is more than probable, since the fragments contained in the granite veins, in the elvans, and in the two systems of mineral veins, exhibit the same concretional forms as those into which the corresponding rocks are now divided by weathering or mechanical action.

Thus I have endeavoured to substantiate my former statement, that the elevatory force could not have acted on a solid mass without the interference of lines of structure; a circumstance which would produce, according to Mr. Hopkins, such considerable modifications in the resulting phænomena, that " to a mass thus constituted these [his physical] investigations must not be considered as generally applicable."

When I commenced this reply, it was my intention to have offered a few remarks on Mr. Hopkins's lengthened comments on the hypothesis which I have advocated concerning the origin of mineral veins in primary districts; but as it is immaterial, in the present case, whether the veins, granite, and slate are or are not all contemporaneous, I think it best not to have our attention diverted from the point at issue, which must be determined by facts, and not by the gratuitous postulata of either hypothesis.

I cannot, however, conclude without again acknowledging the great obligation geologists are under to Mr. Hopkins for his interesting investigations; and, though differing from him on some points, I am not insensible to the great advantages which must accrue to geology in controlling wild speculations by the application of the rigid laws of physical science.

Penzance, Nov. 8, 1836.

V. On the true and extended Interpretation of Formulce in Spherical Trigonometry. By James THomson, LL.D., Professor of Mathematics in the University of Glasgow.*

1. WHILE the rules and theorems given in the moder $\mathbf{n}$ books on trigonometry, in reference to spherical triangles, are sufficient for all practical purposes, yet there are some peculiarities and some curious relations of such triangles, which have either been overlooked in all the works with which I am acquainted, or have been merely glanced at in casual or passing remarks; and hence, as may be expected, some parts of the theory are still imperfectly developed. I shall proceed $\uparrow$

* Communicated by the Author.

+ The formula quoted in this paper will be found in my Elements of Plane and Spherical Trigonometry, and in most of the modern treatises on the subject. 
to give some instances of this kind, and shall commence with the formula

$$
\cos A=\frac{\cos a-\cos b \cos c}{\sin b \sin c},
$$

which enables us to solve the problem in which the three sides are given, to find the angles. Now, since, if $Q$ be any arc or angle, $\cos Q=\cos (2 \pi-Q)$, it is plain, that if $A^{\prime}$ denote one value of the angle $A$, there will be another value $2 \pi-A^{\prime}$, unless it can be shown that this value is excluded for some reason not indicated by the foregoing formula. Let the former of these be less than two right angles; then, if the latter, which we may denote by $A^{\prime \prime}$, be admissible, it will be greater than two right angles *. In like manner the corresponding formulæ would give two expressions, $\mathrm{B}^{\prime}$ and $\mathrm{B}^{\prime \prime}$ $\left(=2 \pi-\mathrm{B}^{\prime}\right)$, for $\mathrm{B}$; and two, $\mathrm{C}^{\prime}$ and $\mathrm{C}^{\prime \prime}\left(=2 \pi-\mathrm{C}^{\prime}\right)$, for $\mathrm{C}$. Now the first of these, $\mathrm{A}^{\prime}, \mathrm{B}^{\prime}, \mathrm{C}^{\prime}$, regarded as being each less than two right angles, are those which are universally, and, in a practical point of view, correctly employed. On consideration, however, we shall find that the latter values are just as admissible as the former. When a great circle is described through two points on the surface of a sphere which are not the extremities of a diameter, the points may be regarded as being joined by either of the arcs into which they divide the circle : it is usual, however, to consider the smaller arc as the one which joins them. Now, if we take on a spherical surface three points which are not on the same great circle, and join them in the way last mentioned, by three arcs, $a, b, c$, we shall obviously divide the entire surface into two parts, each bounded by three arcs of three great circles, and therefore each of them a spherical triangle. The smaller of these is that which is usually alone considered, and its angles are $A^{\prime}, B^{\prime}, C^{\prime}$. The greater has for angles $\mathrm{A}^{\prime \prime}, \mathrm{B}^{\prime \prime}, \mathrm{C}^{\prime \prime}$; and, having $a, b, c$ as sides, it answers the conditions of the problem just as well as the smaller triangle. The angles of the greater triangle are evidently each greater than two right angles.

* It is scarcely necessary to state, that there is no impropriety in regarding an angle as greater than two right angles. If we suppose one radius of a circle to be fixed, and another to revolve from a state of coincidence with it, the motion of the latter will make continual additions to the quantity of angular space described; and the angle made by the lines, commencing from nothing, may be regarded as increasing so as to obtain any magnitude we please. If the line revolve in one direction, the angle may be regarded as positive; if in the other, negative. It is plain also, that if any angle $Q$ has been described, the relative positions of the lines will be the same again after the description, once or oftener, of four right angles in either direction; or, as it may be expressed, the relative positions of the lines will be the same, when the angle which they make is $Q$, as when it $Q \pm 2 n \pi, n$ being a whole number. 
2. What has been thus established affords an explanation of the double sign \pm , before the expressions for the sine and cosine of half any angle of a spherical triangle. Thus, in the formula

$$
\sin \frac{1}{2} A= \pm \sqrt{\frac{\sin (s-b) \sin (s-c)}{\sin b \sin c}}
$$

if we denote the value of $\frac{1}{2} A$ corresponding to the sign + , by $\frac{1}{2} A^{\prime}$, we shall have the other, corresponding to - , equa to $-\frac{1}{2} A^{\prime}$; whence the values of $A$ are $A^{\prime}$ and $-A^{\prime}$; or, by adding $2 \pi$ to the latter, according to the preceding note, the values will be $A^{\prime}$ and $2 \pi-A^{\prime}$. We see, therefore, that the positive value of $\sin \frac{1}{2} A$ gives the value of $\frac{1}{2} A$ in the less of the two triangles bounded by $a, b, c$; and the negative value that of $\frac{1}{2} \mathrm{~A}$ in the greater*.

3. In like manner, in the formula

$$
\cos \frac{1}{2} A= \pm \sqrt{\frac{\sin s \sin (s-a)}{\sin b \sin c}}
$$

the positive and negative values will give respectively $\frac{1}{2} A$ equal to $\frac{1}{2} A^{\prime}$ and $\pi-\frac{1}{2} A^{\prime}$; and consequently $A$ equal to $A^{\prime}$ and $2 \pi-\mathbf{A}^{\prime}$, as before: and, in a similar manner, we may explain the double sign in the formulæ

$$
\tan \frac{1}{2} A= \pm \sqrt{\frac{\sin (s-l) \sin (s-c)}{\sin s \sin (s-a)},}
$$

and $\quad \sin A= \pm \frac{2 \sqrt{\sin s \sin (s-a) \sin (s-b) \sin (s-c)}}{\sin b \sin c}$

4. By considering in a similar manner any of the formulæ which determine the sides by means of the angles, we shall arrive at other results which do not seem to have been hitherto observed. Thus, from the formula

$$
\cos a=\frac{\cos \mathrm{A}+\cos \mathrm{B} \cos \mathrm{C}}{\sin \mathrm{B} \sin \mathrm{C}}
$$

and the corresponding ones for $\cos b$ and $\cos c$, we see that when the three angles are given, each of the sides has two values of the forms $a^{\prime}$ and $2 \pi-a^{\prime}, b^{\prime}$ and $2 \pi-b^{\prime}, c^{\prime}$ and $2 \pi-c^{\prime}$. The values $a^{\prime}, b^{\prime}, c^{\prime}$, each less than a semicircle,

* The same conclusions might be derived from the formula for $\sin \frac{1}{2} A$, taken, not with both its signs, but with either. For, since $\sin Q=\sin (\pi-Q)$, we should have for the value of $\frac{\lambda}{2} A$, corresponding to the positive sign, either $\frac{1}{2} A^{\prime}$ or $\pi-\frac{1}{2} A^{\prime}$; and consequently $A$ equal to $A^{\prime}$ or $2 \pi-A^{\prime}$, as before; while the negative value will give $-\frac{1}{2} A^{\prime}$ and $-\pi+\frac{1}{2} A^{\prime}$; by doubling which, and adding to each $2 \pi$, we get $2 \pi-A^{\prime}$ and $\mathrm{A}^{\prime}$, as before. The same may aloo be done by means of the formula for $\cos \frac{1}{2} \mathrm{~A}$. 
are those which alone are recognised by writers on trigonometry. With regard to the others, they are evidently the remaining arcs of the great circles of which $a^{\prime}, b^{\prime}, c^{\prime}$ are parts, and they are therefore each greater than a semicircle. In fact, if the triangles be clescribed, whose sides are $a^{\prime}, b^{\prime}, c^{\prime}$, and whose angular points are $A, B, C$, so that $A$ and $\mathrm{B}$ are joined by $c^{\prime}, \mathrm{A}$ and $\mathrm{C}$ by $b^{\prime}$, and $\mathrm{B}$ and $\mathrm{C}$ by $a^{\prime}$; then $2 \pi-c^{\prime}$ is the other arc joining $A$ and $B ; 2 \pi-b^{\prime}$ the other arc joining $A$ and $C$, \&c. These larger arcs, being the continuations of $a^{\prime}, b^{\prime}, c^{\prime}$, will evidently form with each other angles which are vertically opposite, and therefore equal, to those made by $a^{\prime}, b^{\prime}, c^{\prime}$. They will therefore answer to the conditions of the problem, since they are arcs of great circles making with each other angles equal to the given angles.

5. It ought to be remarked that $a^{\prime \prime}, b^{\prime \prime}, c^{\prime \prime}$ do not form on the surface of the sphere, a triangle in the ordinary sense of the term: they do not form a space which they bound without intersecting it. The truth is, however, that in both plane and spherical trigonometry, so far as the computation of sides and angles alone is concerned, we have nothing to do with the surface of the triangle, or with any surface whatever; the lengths of lines and their relatize positions being the sole subject of consideration. Thus, when the three sides of a spherical triangle are given, to find the angles, the problem is simply this: Given three points on the surface of a sphere, to find the angles made by the great circles passing through them. So likewise, when the three angles of a spherical triangle are given, to find the sides, the problem, without reference to a triangle, is simply, to find three points on the surface of the sphere, such that the arcs joining them may make with each other angles equal to given angles: and it is easy to see that other problems may be expressed in a similar way; and that the same views, as well as some others in this paper, may also be extended to plane triangles.

6. The problem in which two sides of a spherical triangle, and the contained angle are given, to compute the remaining angles and the third side, may also be viewed in a similar light. Thus, the third side is found by means of the formula

$$
\cos a=\cos \mathrm{A} \sin b \sin c+\cos b \cos c ;
$$

and it is plain, that $a$ will have two values of the forms $a^{\prime}$ and $\left(a^{\prime \prime}=\right) 2 \pi-a^{\prime}$. The smaller is that which is recognised by the writers on trigonometry as the third side; while the other is the remaining arc of the great circle of which $a^{\prime}$ is one part. This will be illustrated by taking on a common globe a point 
above the horizon, and drawing from it two arcs, $b$ and $c$, of great circles to the horizon. Then, if we regard these two, and the angle which they form, as given, we shall have one triangle, which will answer the conditions of the problem, bounded by $b$ and $c$, and the less arc of the horizon joining their extremities; while with the greater or remaining arc of the horizon they will form another triangle, which will answer equally. The latter triangle is evidently composed of the former, together with the hemisphere below the horizon.

7. The remaining angles will be found by the formulæ

$$
\cot B=\frac{\cot b \sin c-\cos A \cos c}{\sin A}
$$

and

$$
\cot \mathrm{C}=\frac{\cot c \cdot \sin b-\cos A \cos b}{\sin A} .
$$

From these, since $\cot Q=\cot (\pi+Q)$, we shall have values for $B$ and $C$ of the forms $B^{\prime}, C^{\prime}$, and $\pi+B^{\prime}, \pi+C^{\prime}$, which evidently correspond to the triangles above mentioned.

8. When a side $a$, and the adjacent angles $\mathrm{B}$ and $\mathrm{C}$, are given, the remaining sides may be found by means of the formulæ

and

$$
\begin{aligned}
& \cot b=\frac{\cot \mathrm{B} \sin \mathrm{C}+\cos \mathrm{C} \cos a}{\sin a} \\
& \cot c=\frac{\cot \mathrm{C} \sin \mathrm{B}+\cos \mathrm{B} \cos a}{\sin a} .
\end{aligned}
$$

These give values for $b$ and $c$, of the forms $b^{\prime}, c^{\prime}$, and $\pi+b^{\prime}$, $\pi+c^{\prime}$; the first of which are the values adopted in the books on trigonometry. The meaning of the others will appear if we enunciate the problem thus: Two great circles being drawn through $\mathrm{B}$ and $\mathrm{C}$, the extremities of a given arc $a$ of a great circle, and making with it given angles; it is required to find the distances between the points $\mathrm{B}$ and $\mathrm{C}$ and the two points of intersection of these circles. These circles will intersect in a point $A$; and if they be continued through that point each to a distance equal to $\pi$, they will again intersect in a point $A^{\prime \prime}$. C A and $B A$ are evidently the arcs $b^{\prime}$ and $c^{\prime}$; while $\mathbf{C} \mathbf{A} \mathbf{A}^{\prime \prime}$ and $\mathbf{B} \mathbf{A} \mathbf{A}^{\prime \prime}$ are respectively $\pi+b^{\prime}$ and $\pi+c^{\prime}$. The third angle comes out, as it ought, of the forms $A^{\prime}$ and $2 \pi-A^{\prime}$, the former being the angle at $A$, and the other the one at $\mathbf{A}^{\prime \prime}$.

9. The case in which there are given a side and the opposite angle, and either another side or another angle, to resolve the triangle, may be treated in a similar manner; but neither case presents any difficulty, and they exhibit nothing remark- 
able in addition to what has been pointed out in the other cases.

10. I shall conclude with some remarks which naturally arise from what has been already saicl.

(1.) Dr. Simson and others define a spherical triangle as being " a figure upon the surface of a sphere comprehended by three arcs of three great circles, each of which is less than a semicircle." The words in italics should be omitted. Analysis, as we have seen above, detects the error, and shows that a side may be of any magnitude not exceeding a complete circle. An author, as the writers on trigonometry have done, may properly confine his attention to triangles having each of their sides less than a semicircle, but he ought not to state that there can be no others.

(2.) The proposition is not true, in which it is asserted, that " the three sides of a spherical triangle are together less than a circle." For a triangle understood in the ordinary meaning, (as bounded by three arcs of great circles, without being intersected by them, ) the limit is two circles, instead of one. To illustrate this familiarly, let us take two points, $\mathrm{A}$ and $\mathrm{B}$, on the horizon of a globe, very near each other, and a third $\mathrm{C}$, nearly diametrically opposite to them, but above the horizon; then draw the smaller of the arcs joining $A C$ and $B C$. By these two arcs, and by the greater arc of the horizon, the sphere will be divided into two triangles, each having one side almost a circle, and each of the others nearly a semicircle.

(3.) Neither is the proposition true, in which it is asserted, that " any two sides of a spherical triangle taken together, are greater than the third side." It is easy to see, by taking one of the sides almost a circle, and the others small, that one side may exceed the sum of the other two in any ratio whatever.

(4.) The assertion is likewise erroneous, that " the sum of the three angles of a spherical triangle cannot be less than two right angles, nor greater than six." The true limits are troo right angles and ten right angles. To illustrate this, let the surface of a sphere be divided into two triangles having extremely small sides. Then, the angles of the smaller triangle being $A, B, C$, those of the other will be $2 \pi-A, 2 \pi-B$, and $2 \pi-C$. The sum of the former will be $\pi+E$, where $\mathbf{E}$ may be as small as we please; while, by adding the others, we find for their sum $6 \pi-(A+B+C)$, or $6 \pi-\pi-E$, or finally, $5 \pi-\mathrm{E}$; that is, ten right angles wanting $\mathrm{E}$.

(5.) The area of a spherical triangle may be of any magnitude between zero and the surface of the sphere.

(6.) The same principles explain some things regarding the 
spherical excess, which seem to have been hitherto overlooked. Thus, $\mathrm{E}$ being the spherical excess, the known formula

$$
\sin \frac{1}{2} \mathrm{E}= \pm \frac{\sqrt{\sin s \sin (s-a) \sin (s-b) \sin (s-c)}}{2 \cos \frac{1}{2} a \cos \frac{1}{2} b \cos \frac{1}{2} c}
$$

gives for $\frac{1}{2} \mathrm{E}$, on account of the double sign \pm , or of the two values belonging to $\sin \frac{1}{2} \mathrm{E}$, or $-\sin \frac{1}{2} \mathrm{E}$, two values, of the form $\frac{1}{2} E^{\prime}$ and $2 \pi-\frac{1}{2} \mathbf{E}^{\prime}$, and, consequently, for $E$ the two $E^{\prime}$ and $4 \pi-E^{\prime}$. Now, the less of these, suppose $E^{\prime}$, answers to the smaller of the two triangles formed by $a, b, c$, and the other to the grenter. For the excess in the former is $\mathrm{A}+\mathrm{B}+\mathrm{C}-\pi$; while in the latter, it is $5 \pi-(\mathrm{A}+\mathrm{B}+\mathrm{C})$, or $5 \pi-\left(\pi+\mathrm{E}^{\prime}\right)$, or, finally, $4 \pi-\mathrm{E}^{\prime}$.

(7.) In like manner, the formula

$$
\cot \frac{1}{2} \mathrm{E}= \pm \frac{1+\cos a+\cos b+\cos c}{2 \sqrt{\sin s \sin (s-a) \sin (s-b) \sin (s-c)}},
$$

gives for $\frac{1}{2} \mathrm{E}$, on account of the double sign, values of the form $\frac{1}{2} \mathrm{E}^{\prime}$, and $2 \pi-\frac{1}{2} \mathrm{E}^{\prime}$. So likewise, from Lhuillier's formula $\tan \frac{1}{4} \mathrm{E}= \pm \sqrt{\tan \frac{1}{2} s \tan \frac{1}{2}(s-a) \tan \frac{1}{2}(s-b) \tan \frac{1}{2}(s-c) \text {, }}$ taking the value of $\tan \frac{1}{4} \mathrm{E}$ first positive and then negative, we find for $\frac{1}{4} \mathrm{E}$ values of the form $\frac{1}{4} \mathrm{E}^{\prime}$, and $\pi-\frac{1}{4} \mathrm{E}^{\prime}$; whence $\mathrm{E}$ will be of the forms $\mathrm{E}^{\prime}$ and $4 \pi-\mathrm{E}^{\prime}$, as before.

(8.) From the formula $\cot \frac{1}{2} \mathrm{E}=\frac{\cot \frac{1}{2} a \cot \frac{1}{2} b+\cos \mathrm{C}}{\sin \mathrm{C}}$, which gives the excess when two sides and the contained angle are the data, we find $\frac{1}{2} \mathrm{E}$ to be of the forms $\frac{1}{2} \mathrm{E}^{\prime}$ and $\pi+\frac{1}{2} \mathrm{E}^{\prime}$; whence the forms of $E^{2}$ will be $E^{\prime}$ and $2 \pi+E^{\prime}$. This answers exactly to the two triangles mentioned in No. 6 ; since the excess $\mathbf{E}^{\prime}$ in the smaller is $\mathbf{A}+\mathbf{B}+\mathbf{C}-\pi$, and in the larger $\mathrm{A}+\pi+\mathrm{B}+\pi+\mathrm{C}-\pi$, or, by contraction, $2 \pi+\mathrm{A}+\mathrm{B}+\mathrm{C}-\pi$, which is the same as $2 \pi+\mathbf{E}^{\prime}$.

Glasgow College, Oct. 21, 1836,

VI. Demonstrations of certain points in Fresnel's Theory of Double Refraction, deduced from the Investigations of the Undulatory Theory which have recently appeared in this Journal. By A Correspondent.

To the Editors of the Philosophical Maguzine and Journal.

\section{Gentlemen,}

DO not know whether the following results are sufficiently original to authorize an expectation that you will be able to afford them a place in your Journal, as, at the most, they 